\title{
Random Matrix Approach to Glassy Physics — Low Temperatures and Beyond
}

\author{
Reimer Kühn and Uta Horstmann \\ Institut für Theoretische Physik, Universität Heidelberg, Philosophenweg 19, 69120 Heidelberg, Germany
}

(Submitted 9 Jan 97)

\begin{abstract}
A random matrix approach to glassy physics is introduced. It leads to a class of models which exhibit both, glassy low-temperature phases, and double- and single-well configurations in their potential energy. The distribution of parameters characterizing the local potential energy configurations can be computed, and differ from those assumed in the standard tunneling model and its variants. Still, low-temperature anomalies characteristic of amorphous systems are reproduced, and we are able to distinguish properties which can be expected to be universal from those which cannot.
\end{abstract}

PACS: 05.20.-y,61.43Fs,64.70.Pf,65.40.+g

Glassy materials exhibit, at low temperatures, a number of properties which are considered anomalous in comparison to those of their crystalline counterparts [1]. Examples are the roughly linear temperature dependence of the specific heat $C(T)$ [2] and the approximately quadratic temperature variation of the thermal conductivity $\kappa(T)$ [3] at $T<1 \mathrm{~K}$, which are in contrast to the $T^{3}$ behaviour of these quantities in crystals. Moreover, between approximately 1 and $20 \mathrm{~K}$, one observes a crossover to a $T^{3}$ behaviour of the specific heat and a plateau in the thermal conductivity. The anomalies below $1 \mathrm{~K}$ appear to be universal in the sense that they are shared by a large variety of amorphous systems, whereas between approximately 1 and $20 \mathrm{~K}$ a stronger dependence on properties of the specific material appears. At still somewhat higher temperatures, a certain degree of universality is again observed, at least in thermal conductivity data [3].

It is the existence of a broad range of localized low energy excitations which is generally held responsible for the aforesaid anomalies. The dominant mechanism giving rise to excitations below $1 \mathrm{~K}$ is believed to be tunneling in double-well potentials (DWP). This is the content of the phenomenological standard tunneling model (STM) 4. A . A different set of excitations in amorphous systems can exist as localized vibrations in soft anharmonic single-well configurations of the potential energy. They have been postulated within the, likewise phenomenological soft-potential model (SPM) [6, ] to describe the physics also above $1 \mathrm{~K}$. Either model requires specific assumptions concerning the distributions of its parameters [4 6] to describe glassy physics at low $T$.

The existence of localized soft vibrations [8], and DWPs [9] responsible for two-level tunneling systems (TLS) has been convincingly established in molecular dynamics studies of glassy systems. In [9], the statistics of the parameters characterizing the local potential energy configurations was also investigated (within the confines of a generalized SPM). Still, as of now it is perhaps safe to characterize both, STM and SPM as providing phenomenological descriptions, based on assumptions which, while plausible in many respects, are still lacking support based on more microscopic approaches, such as that of 11] for $\mathrm{KBr}: \mathrm{KCN}$ mixed crystals. At the same time they are widely used to explain and fit experimental results. In particular, it has been argued [10] that the STM could not really account for the considerable degree of universality observed in glassy physics, and numerical investigations on specific substances [8,9] can at best only partially elucidate this point. It was suggested [10] that universality in glassy systems would emerge only due to sufficiently long-range interactions between TLSs, an idea which has been taken up in [12].

Recently, one of us has proposed a random-matrix model of a glass, which exhibits both, an amorphous low-temperature phase, and a collection of double- (and single-)well configurations in its zero-temperature potential energy surface [13. The main motivation for that study was to formulate a bona-fide model of an amorphous system for which the statistics of local potential energy configurations becomes part of the world of the computable. The result, taken as input for computing the influence of tunnelling-excitations on the lowtemperature properties, was used to demonstrate that the model shares the low-temperature anomalies characteristic of glassy systems. Moreover, it was shown that one could also exhibit relations between low- $T$ and high$T$ phenomena, e.g. between the low-temperature specific heat and the value of the glass-transition temperature itself, and relate this, in turn, with features of the system at the microscopic level of description.

The purpose of the present paper is, inter alia, to investigate the model beyond the two-level approximation in the quantum-mechanical treatment of its local excitations. Thereby we find that it reproduces - without further assumptions - also the characteristic bump as it is observed in $C(T) / T^{3}$ plots of the specific heat. We can trace this back to microscopic features of our model, and we are able to address the universality question posed in [10]: Our answer, in short, is that the ensemble of local potential energies is itself a largely collective affair, and some degree of universality may therefore be expected to arise without interactions at the level of quantized local 
excitations. We shall elaborate on these points below, and also try to situate our model in glassy physics, beyond the specific assumptions embodied in it.

Let us restate the main ingredients of our model. It is based on the following ansatz for the potential energy of $N$ degrees of freedom ("particles") forming a glass-like system,

$$
U_{\text {pot }}(v)=-\frac{1}{2} \sum_{i \neq j} J_{i j} v_{i} v_{j}+\frac{1}{\gamma} \sum_{i} G\left(v_{i}\right),
$$

in which $v_{i}$ may be interpreted as the deviation of the $i$-th particle from some preassigned position. The amorphous aspect is modelled by taking the first, harmonic contribution to $U_{\text {pot }}$ to be random, so that the reference positions would generally turn out to be unstable at the harmonic level of description. A set of anharmonic onsite potentials $G\left(v_{i}\right)$ is therefore added to stabilize the system as a whole. We choose the harmonic part such that the model can be analysed exactly within mean-field theory, and the replica method is used to deal with the disorder. Namely, we take the $J_{i j}$ to be Gaussians with mean $J_{0} / N$ and variance $J^{2} / N$, specializing to $J=1$ to fix the energy-scale. For the on-site potential we choose

$$
G(v)=\frac{1}{2} v^{2}+\frac{a}{4 !} v^{4} .
$$

That is, $G$ also creates a harmonic restoring force, and by varying $\gamma$ we can tune the number of modes in the system which are unstable in the harmonic approximation. Other forms of $G(v)$ may be contemplated; our ability to solve the model does not depend on the particular shape of $G$. The only requirement is that it increases faster than $v^{2}$ for large $|v|$ for the system to be stable, and our choice is the simplest respecting a $Z_{2}$ symmetry.

The choice of random couplings in (1) puts our model outside the class of glass-models in the narrow sense. In view of recent ideas concerning the fundamental similarity between quenched disorder and self-induced disorder as it is observed in glassy systems proper 18, it may still be argued that our choice should capture essential aspects of glassy physics. Models of this type with different couplings and different choices for $G(v)$ have been studied in the context of analogue neuron systems [15,16.

In a mean-field setting, the system is described by an ensemble of effective single-site problems, characterized by potentials which, in replica theory, attain random parameters. Solving the model means to compute the distribution of parameters characterizing the local potential energy configurations self-consistently, and this is precisely what we were afer: it can directly be translated into the distribution of the parameters characterizing DWPs. Taking this distribution as input of a tunneling model, we were able to compute, for instance, the contribution of the tunneling states to the specific heat at low $T$ 13n. In what follows, we shall go beyond the two-level tunneling approximation, and begin to explore the consequences.
To analyze the potential energy surface, one computes the (configurational) free energy of the system

$$
f_{N}(\beta)=-(\beta N)^{-1} \ln \int \prod_{i} d v_{i} \exp \left[-\beta U_{\operatorname{pot}}(v)\right]
$$

and takes its $T=0$ limit to eliminate entropic contributions, and to select one of the system's (many) ground state configurations, using replica theory to average over the disorder so as to get typical results. Standard arguments 14 give $f(\beta)=\lim _{n \rightarrow 0} f_{n}(\beta)$ for the quenched free energy, with

$$
\begin{aligned}
n f_{n}(\beta) & =\frac{1}{2} J_{0} \sum_{a} p_{a}^{2}+\frac{1}{4} \beta \sum_{a, b} q_{a b}^{2} \\
& -\beta^{-1} \ln \int \prod_{a} d v^{a} \exp \left[-\beta U_{\mathrm{eff}}\left(\left\{v^{a}\right\}\right)\right] .
\end{aligned}
$$

Here

$$
U_{\mathrm{eff}}=-J_{0} \sum_{a} p_{a} v^{a}-\frac{\beta}{2} \sum_{a, b} q_{a b} v^{a} v^{b}+\frac{1}{\gamma} \sum_{a} G\left(v^{a}\right)
$$

is a replicated single-site potential and the order parameters $p_{a}=N^{-1} \sum_{i}\left\langle v_{i}^{a}\right\rangle$ and $q_{a b}=N^{-1} \sum_{i}\left\langle v_{i}^{a} v_{i}^{b}\right\rangle$ are determined from the fixed point equations

$$
\begin{aligned}
p_{a} & =\left\langle v^{a}\right\rangle, a=1, \ldots, n \\
q_{a b} & =\left\langle v^{a} v^{b}\right\rangle \quad, a, b=1, \ldots, n,
\end{aligned}
$$

in which $\langle\ldots\rangle$ denotes a Gibbs average corresponding to the replica potential (5), and the limit $n \rightarrow 0$ is eventually to be taken.

We have evaluated (4)-(7) in the replica symmetric (RS) 13] and the 1st step replica-symmetry breaking (1RSB) approximations. In RS one assumes $p_{a}=p$ for the 'polarization', and $q_{a a}=\hat{q}$ and $q_{a b}=q$ for $a \neq b$ for the diagonal and off-diagonal entries of the EdwardsAnderson matrix. These are determined from

$$
p=\langle\langle v\rangle\rangle_{z}, \quad C=\left\langle\frac{1}{\sqrt{q}} \frac{d\langle v\rangle}{d z}\right\rangle_{z}, \quad q=\left\langle\langle v\rangle^{2}\right\rangle_{z} .
$$

Here $C=\beta(\hat{q}-q)$, and $\langle\ldots\rangle_{z}$ denotes an average over a standard Gaussian $z$ while $\langle\ldots\rangle$ is now a Gibbs average corresponding to the RS single-site potential

$$
U_{\mathrm{eff}}(v)=-h_{\mathrm{RS}} v-\frac{1}{2} C v^{2}+\frac{1}{\gamma} G(v),
$$

with $h_{\mathrm{RS}}=J_{0} p+\sqrt{q} z$. The $\mathrm{RS}$ approximation thus describes a Gaussian ensemble of single-site potentials $U_{\text {eff }}(v)$, with parameters $p, q$ and $C$ which are determined self-consistently through (8).

The system described by (8)-(9) exhibits a transition from an ergodic phase with $p=q=0$ to a glassy phase with $q \neq 0$ at some temperature $T_{g}$ depending on $J_{0}$ and $\gamma$; see Fig. 1. If $J_{0}$ is sufficiently large, a transition 
to a polarized phase with $p \neq 0$ may also occur. The assumption of RS is not always correct. Replica symmetry breaking (RSB) occurs at low temperatures and large $\gamma$. The location of the instability against RSB is given by the AT criterion [17] $1=\beta^{2}\left\langle\left(\left\langle v^{2}\right\rangle-\langle v\rangle^{2}\right)^{2}\right\rangle_{z}$. In the $1 \mathrm{RSB}$ approximation, which is expected to constitute a major step towards the full solution, $U_{\text {eff }}(v)$ is of the same form $(9)$, albeit with $h_{\mathrm{RS}}$ replaced by $h_{1 \mathrm{RSB}}=$ $J_{0} p+\sqrt{q_{0}} z_{0}+\sqrt{q_{1}-q_{0}} z_{1}$, and $C$ by $C=\beta\left(\hat{q}-q_{1}\right)$, where we use standard notation 14 for the entries of the $q$-matrix. Along with a so-called partitioning parameter $m$, they are determined from a more complicated set of fixed point equations [14,19. Now $z_{0}$ is a Gaussian, whereas the distribution of $z_{1}$ is more complicated.
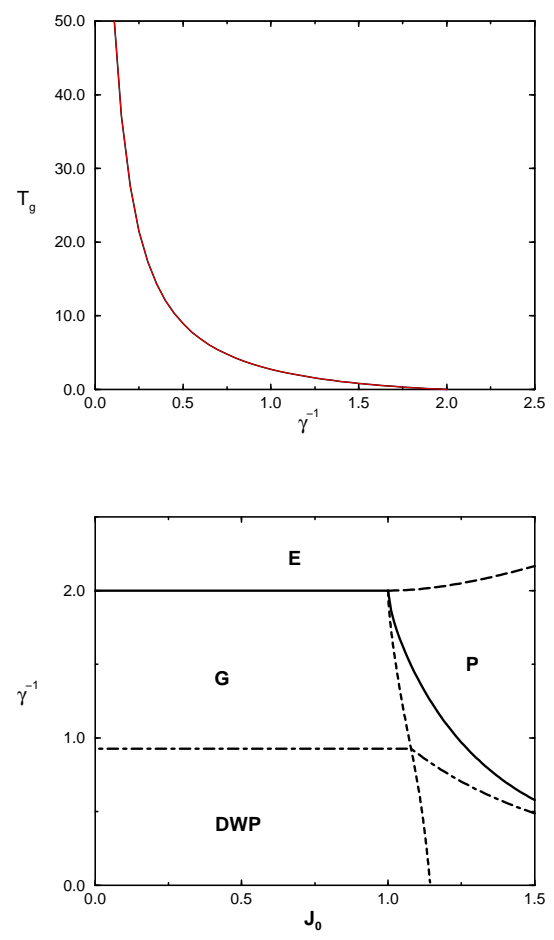

FIG. 1. (a): Glass temperature $T_{g}$ as a function of $\gamma$ for $J_{0}=0$. (b): $T=0$ phase diagram in RS. $E$ denotes the $T=0$-limit of the ergodic phase, $G$ the glassy phase, and $P$ a phase with macroscopic polarization. The full line is the AT line. Below the dot-dashed line is the region with DWPs. The short-dashed line separates the glassy phase $G$ from the phase $P$ with macroscopic polarization.

Interestingly, the system can also exhibit a collection of DWPs in its $T=0$ potential energy surface. That is, for suitable external parameters, some of the $U_{\text {eff }}(v)$ have DWP form. From (2),(9) it is clear that the condition for this to occur is $\gamma C>1$; this region is marked DWP in Fig. 1b. DWPs exist for not too large $\left|h_{\mathrm{RS} / 1 \mathrm{RSB}}\right|$, and the distribution of their characteristic parameters derives from the Gaussian nature of $z$ (the joint $\left(z_{0}, z_{1}\right)$ distribution in the $1 \mathrm{RSB}$ case) and can be computed.
These are barrier height $V$, asymmetry $\Delta$, distance $d$ between the wells, and the tunnelling matrix element $\Delta_{0}=\hbar \omega_{0} \exp (-\lambda)$, with $\lambda=d\left(2 m_{0} V / \hbar^{2}\right)^{1 / 2}, \omega_{0}$ a characteristic frequency (of the order of the frequency of harmonic oscillations in the two wells forming the DWP) and $m_{0}$ the effective mass of the tunneling particle. For larger $\left|h_{\mathrm{RS} / 1 \mathrm{RSB}}\right|$, the $U_{\mathrm{eff}}(v)$ only exhibit single-well forms.

In contrast to the main assumption of the STM that $\Delta$ and $\lambda$ are uncorrelated with $P(\Delta, \lambda) \simeq P_{0}$, we find $\Delta$ and $\lambda$ to be strongly correlated; both are functions of one random variable $h_{\mathrm{RS} / 1 \mathrm{RSB}}$ in RS/1RSB respectively, a feature which persists at all finite levels of RSB. By the same token, SPM expansion-coefficients of $U_{\text {eff }}(v)$ are correlated. The correlations between $\lambda$ and $\Delta$ and likewise those between the SPM coefficients can be weakened (but not eliminated) by introducing local randomness, i.e., by making either $\gamma$ or other parameters of the onsite potentials $i$-dependent in a random fashion. It has been demonstrated in [16] that our model remains solvable with these modifications. The RS distributions $P(\lambda)$ and $P(\Delta)$ have been presented in [13]. Both have singularities at their upper boundary, the former an integrable divergence, the latter a cusp singularity.

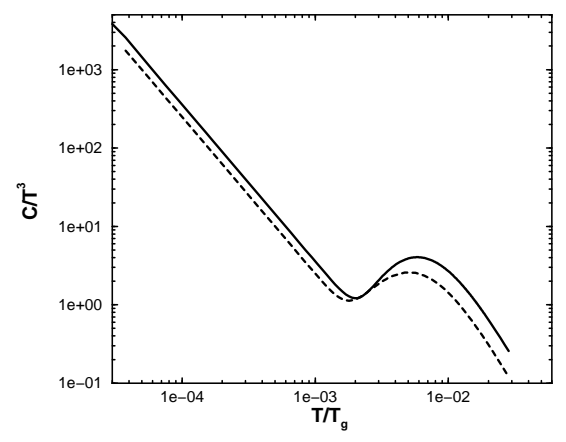

FIG. 2. Low $T$ specific heat in the $G$-phase for $\gamma=10$, and $J_{0}=0$ in the RS (full line) and $1 \mathrm{RSB}$ (broken line) approximation. Note that the location of the bump at $T_{b} \simeq 6 \times 10^{-3} T_{g}$ is reasonable for many glasses. E.g. for a- $\mathrm{SiO}_{2}$ this would correspond to $T_{b} \simeq 8.6 \mathrm{~K}$.

Even though $P(\Delta, \lambda)$ is different from what is assumed in the STM, we find that the contribution of the tunneling excitations to the specific heat exhibits an extended range of temperatures where it scales linearly with $T$. By solving the Schrödinger equation of a particle moving in the potential $U_{\text {eff }}(v)$ and by computing the specific heat beyond the two-level approximation, we obtain a bump in a $C(T) / T^{3}$-plot (Fig. 2), as it is typically also observed in experiments. Its microscopic origin is related to "harmonic" excitations in DWP and SWP structures whose frequency depends only weakly on $h_{\mathrm{RS} / 1 \mathrm{RSB}}$, thus $\Delta$, and which gives rise to a peak in the density of states (DOS). For DWPs with larger asymmetries, the role of 
tunneling is, indeed, mainly to prevent level crosssings of these "harmonic" excitations as $\Delta$ is varied.

Within our model there is a clear distinction between the local $G\left(v_{i}\right)$ contributions to $U_{\text {pot }}$ and the global ones mediated by the $J_{i j}$. It is the influence of the latter which makes the ensemble of local potential energy configurations a collective affair and which can thus be regarded as a source of universality of glassy low-temperature physics. The effect of the $J_{i j}$ on the $U_{\text {eff }}\left(v_{i}\right)$ is to produce a linear term $-h_{\mathrm{RS} / 1 \mathrm{RSB}} v$, which creates a broad range of asymmetries and thereby a nearly constant contribution to the DOS at low energies, and a renormalization of the harmonic contribution through $-\frac{1}{2} C v^{2}$, which is responsible for the very occurrence of DWPs. Conversely, local properties embodied in $G(v)$ are mainly responsible for the "harmonic" excitations creating peaks in the DOS on top of the unstructured part. These cannot be expected to be universal, which fits rather nicely with the experimental observation that both the bump in the $C(T) / T^{3}$ plot and the corresponding plateau in $\kappa(T)$ (presumably created by resonant scattering of phonons from these harmonic excitations) do show a stronger material dependence. It is not unreasonable to assume that such a distinction between local and global contributions to $U_{\text {pot }}$ can be made for real materials as well (perhaps with randomness in the local contribution), the long-range global one being, e.g., of elastic origin. Interestingly, on introducing local randomness such as non-degenerate distributions for the $\gamma$, we have obtained slightly super-linear low- $\mathrm{T}$ specific heats, with exponents ranging between 1 and approximatly 1.5 [19], which fits nicely with experimental observations. It is currently under systematic study. As mentioned above, local randomness will also weaken the perfect correlation between $\lambda$ and $\Delta$ which is also desirable in view of the experimental situation 1 .

From the nearly constant DOS at low energies we can determine the constant $P_{0}$ [i]. For parameters as in Fig. 2, we find $P_{0} \simeq 6 \cdot 10^{-3}\left(k_{\mathrm{B}} K\right)^{-1} /$ atom in RS (aproximately half this value in $1 \mathrm{RSB}$ ), which is roughly three orders of magnitude too large for most glasses. While this may be regarded as a deficiency, we believe that it does not invalidate our approach (no tuning of parameters whatsoever has yet been attempted) but rather that it may be used to guide further detailed modelling: again, local randomness turns out to be a possible cure.

We have proposed and solved a simple model which exhibits both, an amorphous low-temperature phase, and a collection of DWPs and SWPs in its potential energy landscape, and which reproduces characteristic glassy low temperature anomalies, though, as a model based on localized degrees of freedom it cannot be expected to present a faithful picture in all details of the ergodic (liquid) phase. Also, we do not presently regard our model as providing a realistic description of specific substances but rather as representative for a new and promising way of looking at glassy physics - at low temperatures and beyond. For instance, models of the type proposed will also exhibit interesting dynamical properties at or near their glass temperature $T_{g}$ and possibly throughout their glassy phase, which might be investigated, e.g., along the lines of 20]. Finally, we believe that our approach does hold the potential for eventually including realism; the search for good models has only just begun.

We would like to thank C. Enss, H. Horner, S. Hunklinger, J. van Mourik, P. Neu, O. Terzidis, J. Urmann, and $\mathrm{A}$. Würger for numerous illuminating discussions.

[1] for reviews, see S. Hunklinger and W. Arnold, in Physical Acoustics, edited by W.P. Mason and R.N. Thurston (Academic, New York, 1976) Vol. XII, p. 155; W. A. Phillips, Rep. Progr. Phys. 50, 1675 (1987)

[2] R.C. Zeller and R.O. Pohl, Phys. Rev. B 4, 2029 (1971)

[3] J.J. Freeman and A.C. Anderson, Phys. Rev. B 34, 5684 (1986)

[4] P.W. Anderson, B.I. Halperin, and C.M. Varma, Phil. Mag. 25, 1 (1972)

[5] W.A. Phillips, J. Low Temp. Phys. 7, 351 (1972)

[6] V.G. Karpov, M.I. Klinger, and F.N. Ignat'ev, Sovj. Phys. JETP 57, 439 (1983)

[7] U. Buchenau, Yu.M. Galperin, V.L. Gurevich, D.A. Parshin, M.A. Ramos, and H.R. Schober, Phys. Rev. B 46, 2798 (1992)

[8] H.R. Schober and B.B. Laird, Phys. Rev. B 44, 6746 (1991)

[9] A. Heuer and R.J. Silbey, Phys. Rev. Lett. 70, 3911 (1993)

[10] C.C. Yu and A.J. Leggett, Comments Cond. Mat. Phys. 14, 231 (1988)

[11] E.R. Grannan, M. Randeira, and J.P. Sethna, Phys. Rev. Lett. 60, 1402 (1988)

[12] A.L. Burin and Yu. Kagan, JETP 82, 159 (1996), and references therein

[13] R.Kühn, in: Complex Behaviour of Glassy Systems, Proceedings of the XIVth Sitges Conference, edited by M. Rubi, Springer Lecture Notes in Physics (Springer, Berlin, Heidelberg, 1996)

[14] M. Mézard, G. Parisi, and M. A. Virasoro, Spin Glass Theory and Beyond, (World Scientific, Singapore, 1987)

[15] J. Hopfield, Proc. Natl. Acad. Sci. USA, 81, 3088 (1984)

[16] R. Kühn, S. Bös, and J.L. van Hemmen, Phys. Rev. A 43, 2084 (1991); R. Kühn and S. Bös, J. Phys. A 26831 (1993)

[17] J.R.L. de Almeida and D.J. Thouless, J. Phys. A 11, 983 (1978)

[18] J.P. Bouchaud, L. Cugiandolo, J. Kurchan, and M. Mézard, Physica A226, 243 (1996), and references therein

[19] U. Horstmann and R. Kühn, in preparation

[20] H. Sompolinsky and A. Zippelius, Phys. Rev. B 25, 6860 (1982) 